\title{
Reserva de la Biosfera de los Ancares Leoneses: laboratorio de participación social en la gestión del patrimonio
}

Nuria Alonso Leal | gerente de la Reserva de la Biosfera de los Ancares Leoneses Pedro M. ${ }^{a}$ Herrera Calvo | Gama SL, asesoría técnica de la Reserva de la Biosfera de los Ancares Leoneses

URL de la contribución <www.iaph.es/revistaph/index.php/revistaph/article/view/3408>

\section{RESUMEN}

La reserva lleva inmersa desde principios del año 2011 en un proceso de reactivación cuya principal característica es la participación de la población local tanto en la toma de decisiones como en las actuaciones ligadas a la planificación y gestión del territorio. Desde que se inició el proceso de reactivación de la reserva de la biosfera de los Ancares Leoneses, sus responsables han entendido que la población local es la protagonista del territorio incluido en esta denominación, como debería serlo quizá en el conjunto del medio rural. Sus habitantes son los principales herederos de un patrimonio que sus antepasados conservaron hasta la actualidad y les legaron, y los que disfrutan y sufren, en primera persona, las peculiaridades del entorno que habitan. Esta cuestión se ha considerado una premisa de partida a la hora de plantear las actividades a desarrollar en la zona así como a la de poner en funcionamiento los instrumentos de gestión de la Reserva de la Biosfera de los Ancares Leoneses (RBALE) y sus documentos estratégicos. La participación local se convierte en la espina dorsal de la metodología y el funcionamiento de la reserva.

\section{Palabras clave}

Ancares (España, Sierra) | Reserva de la Biosfera de los Ancares Leoneses | Reservas de la biosfera | España, León (Provincia) | Patrimonio natural | Planificación estratégica | 


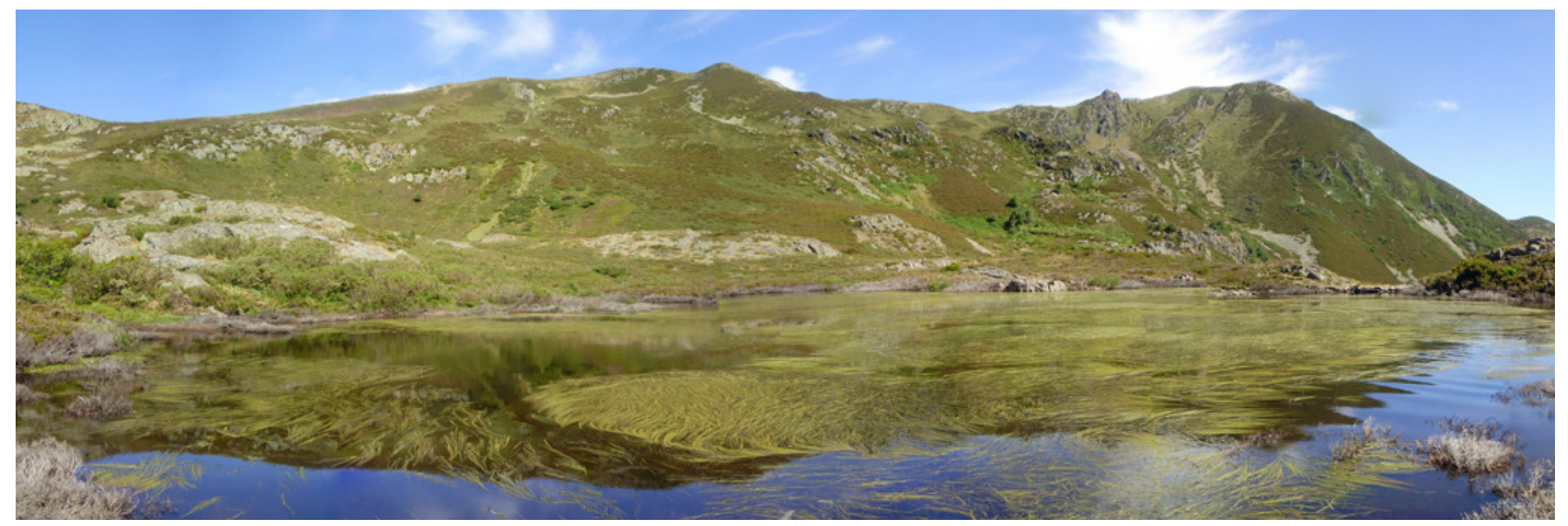

Lagos de Burbia | foto RBALE (Susana Abad)

\section{ANCARES LEONESES, EL CORAZÓN DE LA MONTAÑA BERCIANA}

La comarca leonesa de Ancares es un área de montaña localizada al norte de la provincia de León, en su engarce con Asturias y Galicia. Ocupa, por tanto, la vertiente sur de la cordillera cantábrica, un paisaje montañoso, ganadero y profundamente transformado, en el que el Mediterráneo va desplazando poco a poco los climas y paisajes húmedos del norte a medida que descendemos hacia la depresión del Bierzo.

La comarca ancareña incluye una serie de valles glaciares paralelos que en la vertiente leonesa discurren de noroeste a sureste; son los valles de Fornela, Ancares, Burbia y Porcarizas, que enlazan con la Olla del Bierzo a través de los viñedos y frutales, el municipio de Villafranca del Bierzo, que también forma parte de la reserva de la biosfera, junto con los de Candín, Peranzanes y Vega de Espinareda.

El patrimonio natural y cultural de estas tierras es abrumador. Desde los paisajes glaciares de alta montaña a los encinares mediterráneos, y desde los pastizales alpinos a los abedulares y rebollares, los hábitats de Ancares acogen una comunidad ecológica compleja y valiosa, con especies emblemáticas como el oso pardo y otras menos conocidas pero muy interesantes como la perdiz pardilla o la liebre de piornal. A su importancia ecológica y paisajística se une un patrimonio cultural muy destacado. Por ejemplo, en cuanto al patrimonio construido, resaltan los molinos y otras edificaciones vinculadas al aprovechamiento hidráulico y, sobre todo, las pallozas, la construcción típica ancareña de teito de paja de centeno que se compartía con los animales domésticos. Y no debe olvidarse un patrimonio inmaterial abundantísimo, con manifestaciones culturales que se pierden en el tiempo como las danzas fornelas o la extensa cultura ganadera que aún puede apreciarse en muchos de sus pueblos. 


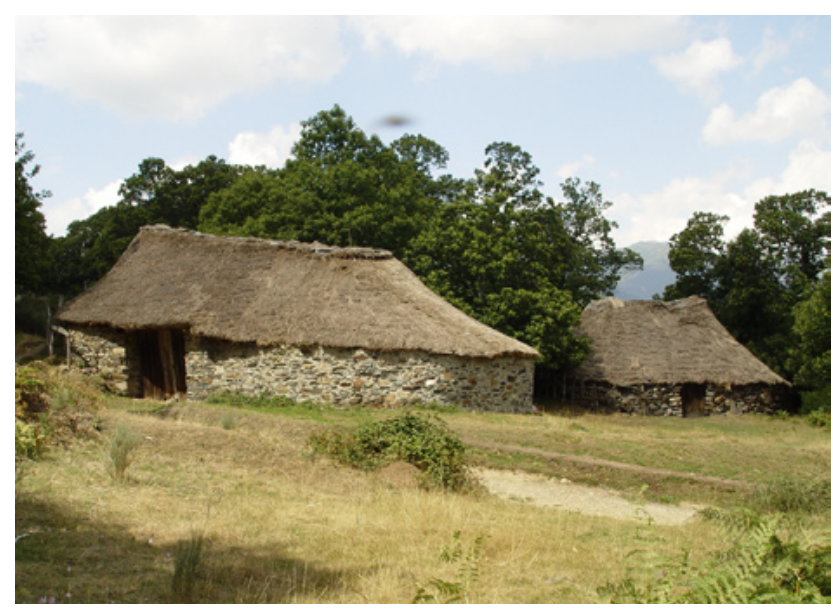

En el año 2006, los Ancares Leoneses fueron declarados por la UNESCO como reserva de la biosfera, designación que une, a través del programa MaB (Man and Biosphere, Persona y Biosfera), a aquellos territorios especialmente valiosos en cuanto a su patrimonio social, natural y cultural.

Este patrimonio constituye una herencia creada y conservada desde su propio interior, imbricada en una compleja red de relaciones que agrupa a los distintos elementos del territorio, incluidas las personas. Una cuestión clave en la conservación de estos valores es, precisamente, el papel que debe jugar su propia población. ¿Quién debe ser el protagonista de la gestión del territorio? El modelo planteado por los Espacios Naturales Protegidos tradicionales, organizado desde los gobiernos regionales y con una mínima intervención, no sólo de la población, sino también de la política local, no está dando los frutos esperados. Por su parte, los ayuntamientos tampoco poseen los medios técnicos o los recursos necesarios para hacer frente a una tarea tan compleja como ésta, ni, por supuesto, las competencias adecuadas. Finalmente, tampoco las instituciones académicas ni, lo que resulta cuando menos sorprendente, la propia población han podido participar en la definición de las sucesivas políticas vinculadas a la conservación de su patrimonio. Eso sí, son los principales destinatarios de todas las medidas que se proponen, las de estímulo y las coercitivas, las buenas y las malas. Y todas esas iniciativas que vienen y van y que la gente de los pueblos ven pasar $y$ desaparecer mientras ellos siguen todavía allí.

Con una visión más integral de la complejidad del paisaje y las relaciones sociales, económicas, culturales y ecológicas del territorio, posiblemente la figura reserva de la biosfera es la que mejor invita a desarrollar un modelo de gestión compartida. El planteamiento de la reserva de los Ancares Leoneses se apoya en tres pilares básicos: los representantes locales, estatales e internacionales de las administraciones públicas, los técnicos y científicos,
Pallozas de Sorbeira | foto Javier Pérez Gil Balouta (2013) | foto Rodrigo Garnelo 
y la población local. La figura de reserva de la biosfera está llamada, desde nuestro punto de vista, a servir como nexo de unión, facilitador e impulsor de la apertura de nuevas vías de diálogo y cooperación entre las diferentes partes.

Posiblemente, no es necesario mencionar la relación directa entre el abandono del medio rural que sufre el interior de nuestro país y el deterioro de la gestión del patrimonio. Poco a poco, la conservación y mejora del patrimonio se va dejando descansar en manos competentes pero ajenas, lo que produce una desconexión entre las necesidades reales y las actuaciones llevadas a cabo. Así, lo que debería ser una intervención continua y compleja queda reducida, en gran parte de las ocasiones, a proyectos financiados externamente, de duración limitada, que terminan sin conseguir una masa crítica que los sostenga y acaban por contribuir a la situación de despoblación y al efecto dominó que genera la pérdida de recursos.

El objetivo de las más de 600 reservas de la biosfera de todo el mundo es funcionar como factorías de ideas, buscando un modelo de desarrollo alternativo y equilibrado. Están llamadas a convertirse en auténticos "laboratorios de sostenibilidad", integrando sus tres funciones principales: la conservación de los recursos naturales y culturales, el desarrollo humano y socioeconómico y el apoyo logístico a la investigación, el aprendizaje, el intercambio y la comunicación.

Así, para un territorio concreto, el hecho de ser declarado como reserva de la biosfera, más allá de la distinción que se le otorga, debe convertirse en una plataforma de oportunidad, que puede manifestarse en muchos sentidos: desde ofrecer un modelo de planificación participada, plantear estrategias de desarrollo compatible con la conservación o ser altavoz de un modo de vida y de generación de riqueza que va mas allá de lo meramente económico. Y, sin duda, es una oportunidad para gestionar el patrimonio de una forma poco habitual en los tiempos que corren, a pesar de las palabras, los planes, las ideas y las intenciones que tan a menudo se oyen y tan difícilmente llegan a plasmarse en la realidad.

La propia definición de "reserva de la biosfera" lleva aparejada, de forma inherente, una implicación de la población con todo lo que tiene que ver con el manejo y aprovechamiento del medio y sus recursos. $Y$ aunque no siempre es fácil poner en marcha la maquinaria necesaria para realizar esta tarea, en la Reserva de la Biosfera de los Ancares Leoneses (en proceso de reactivación desde el año 2011 gracias al impulso técnico aportado por la Fundación Ciudad de la Energía), ése es el horizonte. Así, sus responsables han entendido que la población local es la protagonista de su territorio, como debería serlo en el conjunto del medio rural. Se entiende que los habitantes han recibido como legado el patrimonio que sus antepasados conservaron 
mejor o peor hasta la actualidad y ellos son los que sufren y disfrutan, en primera persona, las peculiaridades del lugar en el que habitan. Esta cuestión se ha considerado una premisa básica de cara a proponer actuaciones en la zona así como a la hora de poner en funcionamiento los instrumentos de planificación y gestión de la Reserva de la Biosfera de los Ancares Leoneses (RBALE). La participación local se ha constituido en la espina dorsal de la metodología y el funcionamiento de la reserva.

El carácter innovador de esta manera de trabajar es limitado ya que a lo largo y ancho de la geografía mundial son numerosas las iniciativas que han tratado y tratan de poner en valor el capital social local como bandera de un trabajo de desarrollo sostenible. Entre ellas, la custodia del territorio aglutina diversas técnicas de responsabilidad compartida por diferentes agentes en tareas de conservación; también las agendas locales 21, tan populares en su momento, plantean otra escala de participación al planificar la estrategia de un municipio de acuerdo a las necesidades del planeta. Sin embargo, el experimento que se quiere probar en este laboratorio natural que es Ancares Leoneses no es sólo planificar entre todos y no es sólo decidir entre todos. Busca poder desarrollar entre todos estas decisiones consensuadas. Y lo más importante, que esta metodología se mantenga en el tiempo y sea capaz de evolucionar y madurar hacia un modelo auténticamente democrático y participativo de gestión territorial. No es fácil, no es habitual, pero tampoco hemos encontrado otra alternativa mejor ni que tenga el éxito garantizado.

\section{METODOLOGÍA DE LA PARTICIPACIÓN EN LA RBALE}

La participación social en la gestión del territorio es un proceso y como tal hay que entenderlo a la hora de planificar y generar expectativas. La descripción metodológica de este modelo de gestión territorial, a partir de fases estancas, es sólo una manera de ordenar ideas y asegurarse de que no quede nada en el tintero, pues es obvio que el proceso de planificación y gestión de actuaciones es una cuestión no sólo de carácter continuo, sino profundamente entrelazada, en la que el desarrollo de una tarea lleva implícito el nacimiento de otras muchas y se produce una retroalimentación constante. Se ha tratado, no obstante, de organizar y separar las propuestas a partir de la división en pilares establecida en la introducción, desarrollando los aspectos referidos a la población local, a la comunidad científica y a las administraciones.

\section{Primer pilar: población local}

Una de las tareas más difíciles de la participación es la de llegar a un territorio determinado (con un bagaje personal y de equipo y un encargo concreto 
como gestores) y tratar de crear una dinámica que suponga la implicación de los habitantes de toda esa zona. No se trata de convocar a las poblaciones locales a acudir a actividades ya organizadas, sino de atraer a un grupo grande y representativo de personas (de muy diversas procedencias, capacidades e ideologías) y convencerlas de que aporten su tiempo, su trabajo y su experiencia para decidir qué se debe hacer, estableciendo las prioridades y liderando las actuaciones. Ése es el significado real de la participación en la toma de decisiones. El planteamiento es el siguiente: no significa únicamente consultar, aunque se consulte a todos los implicados; no significa informar, aunque sea necesario disponer de una información fiable y veraz; ni significa invitar a algo que ya está establecido, aunque ser una reserva de la biosfera implica asumir una serie de obligaciones. Participar significa adoptar un marco de trabajo compartido, unos objetivos comunes y unas limitaciones que parten de la propia figura (una reserva de la biosfera no tiene competencias en la gestión del territorio aunque debe demostrar la compatibilidad de las personas con la conservación del patrimonio, tampoco dispone de un presupuesto asignado para desarrollar actividades, aunque a cambio tiene un ámbito mucho más amplio y puede plantearse ser muchas cosas diferentes). El comienzo de la participación implica preguntar a la gente qué es lo que quiere para su territorio e involucrarla en conseguir esas metas.

Este proceso de participación necesita, además, una capacitación progresiva de sus actores. El equipo técnico está desde el principio a disposición de los participantes, funcionando como un espejo que refleja una imagen de la realidad del territorio y sus habitantes, transmitiendo lo que significa ser reserva de la biosfera y, al mismo tiempo, catalizando la construcción de una red de trabajo, tiempo, energía e ideas capaz de avanzar en la dirección correcta. Este es el reto asumido por la RBALE que, con sus dificultades, sus errores y sus fracasos, siendo consciente de que no existe una fórmula mágica para generar procesos de autogestión cuando estos no son endógenos, pretende buscar una fórmula propia del territorio.

Esta cesión de la capacidad de decidir implica que la información debe ser recopilada y organizada de forma mucho más cuidadosa y asequible para los participantes. Además, la tutela de un proceso de este tipo demanda una gran habilidad por parte de los dinamizadores y mediadores para facilitar la toma de decisiones y la adopción de acuerdos sin interferir en el resultado final, implicándose en la dinámica del proceso y en la calidad de sus resultados pero no en el contenido de éstos.

Elaboración del Plan Estratégico y Plan de Acción 2011-2013. Hacia una estrategia participada de desarrollo sostenible

Las directrices de las reservas de la biosfera, recogidas en los planes de acción de Madrid y del Montseny, de carácter internacional y nacional res- 


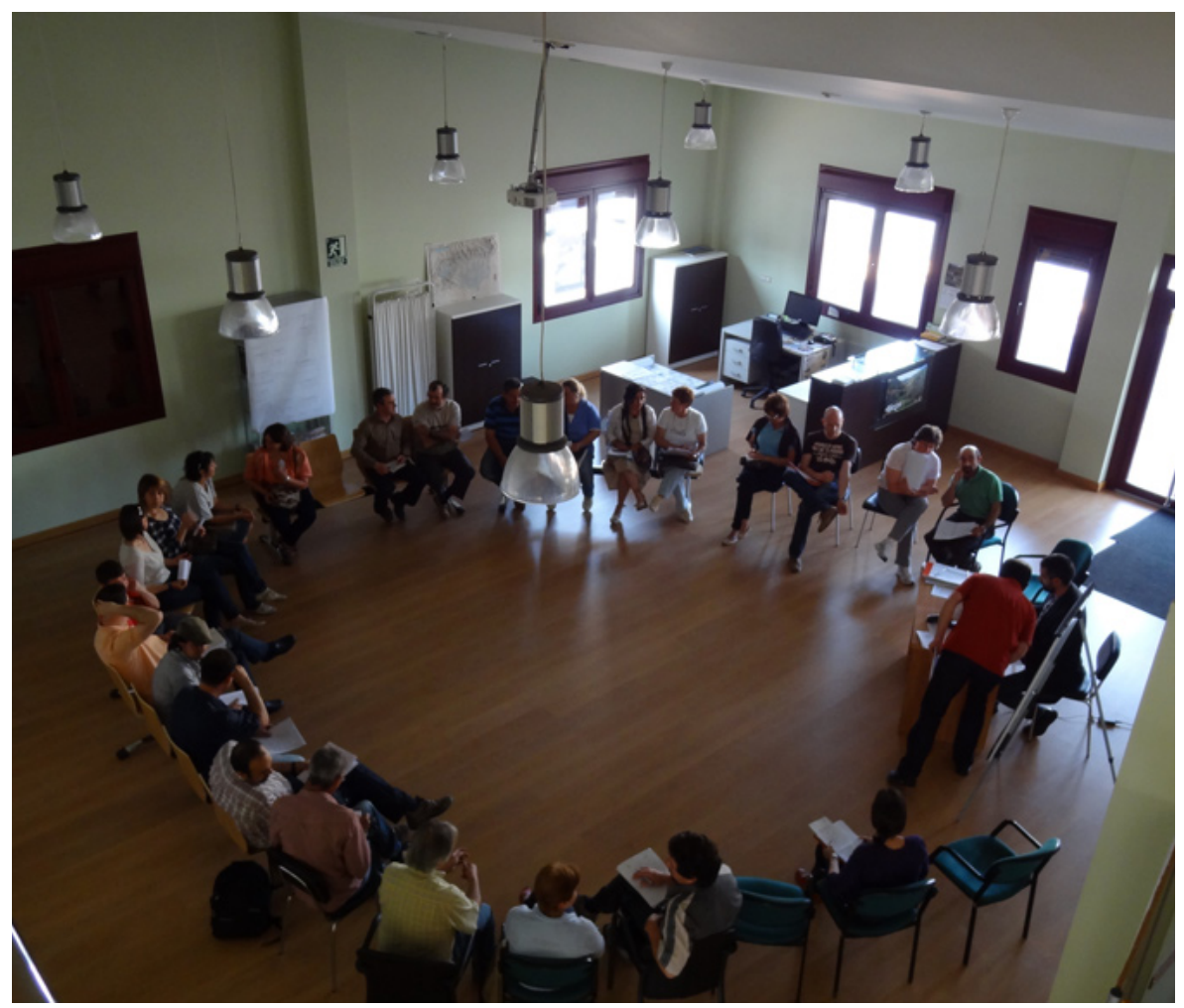

Miembros del Consejo de Participación de la Reserva de la Biosfera de los Ancares Leoneses | foto RBALE

pectivamente, marcan que cada reserva debe disponer de una serie de instrumentos estratégicos, incluyendo un plan de acción con un marco temporal predeterminado. Una vez decididos a reactivar la RBALE, resultaba imprescindible, en primer lugar, la redacción y aprobación de este instrumento. Ya desde el principio se decidió trabajar en dos textos diferentes: un plan estratégico que planteara objetivos y metas generales así como una línea estratégica para llegar a ellos; y un plan de acción, destinado a concretar actuaciones, plazos y recursos necesarios para llevarlas a cabo. El reto de hacerlo incluyendo a la población en la toma de decisiones fue una iniciativa propia de los gestores de la reserva.

La elaboración de estos documentos se planteó a partir de un proceso de participación desarrollado en tres fases sucesivas siguiendo una propuesta de marco lógico, que podemos dividir en: organización del trabajo, información y diagnóstico, y redacción de las actuaciones del plan.

- Fase de organización del trabajo

El trabajo comienza con dos tareas simultáneas. Por un lado, se programa y organiza el proceso, adoptando un calendario base para su desarrollo; y, por 
otro, se organiza toda la información disponible sobre el territorio que pueda ser de utilidad de cara a las fases sucesivas estableciéndose los contactos necesarios con los agentes activos en el territorio.

- Fase de diagnóstico

Esta fase se inicia con la actualización de un diagnóstico técnico que recoge las características físicas, ecológicas y socioeconómicas de la RBALE. A continuación se realiza el diagnóstico propiamente dicho, procesando esta información, estudiando las fortalezas y debilidades y planteando, además, algunas orientaciones para la acción. No obstante, de cara al trabajo en el territorio e independientemente de la amplitud y profundidad de la información existente, resulta de suma importancia conocer la percepción de los problemas y de la realidad que experimenta la propia población. La visión que tienen los habitantes del territorio y su situación actual resulta tan importante o más que la información técnica, de cara a desarrollar unos instrumentos de planificación realmente centrados en su realidad.

Este análisis, denominado diagnóstico participado o social, se elabora a partir de una intensa recopilación de las opiniones y las visiones locales.

Desde esta visión subjetiva se plantean también una serie de actividades de diagnóstico utilizando técnicas específicas, como las matrices DAFO (debilidades, amenazas, fortalezas y oportunidades), los árboles de problemas y otras herramientas. La interpretación de estas actividades de análisis permite, a su vez, ir planteando las líneas básicas de mejora y se van definiendo y discutiendo propuestas e ideas. El diagnóstico social incorpora, de esta manera, una primera recopilación de líneas y propuestas de actuación.

El diagnóstico social sirve también como punto de encuentro y como un ámbito de formación y capacitación entre los propios participantes de cara al proceso de redacción de los planes. Sin embargo, es importante no perder el contacto con el resto de la población local y continuar trabajando para incorporar nuevas visiones y participantes, desarrollando algunas actividades informativas y de divulgación, al amparo del ya incipiente programa de comunicación de la RBALE. El objetivo era acercar una propuesta de actividades inspiradoras para que los destinatarios fueran tomando conciencia de su papel activo en la reserva, incluyendo algunas visitas "estratégicas", una exposición sobre reservas de la biosfera con motivo del 40 aniversario del Programa MaB y una visita a la reserva de la biosfera de las Sierras de Béjar y Francia que nos acercara a una realidad similar en pleno funcionamiento.

Finalmente, ambas visiones se integran en un diagnóstico estratégico, que cruza las aportaciones sociales con la base técnica y establece un punto de partida para debatir la estrategia de desarrollo de la RBALE. 


\section{- Fase de propuesta}

Esta última fase, una vez concluidas las actividades formativas e informativas y elaborados ya los documentos de diagnóstico, se concreta en la redacción de los documentos estratégicos. En este caso resulta muy importante la secuencia de las dinámicas programadas ya que de su correcto planteamiento y ejecución depende, en gran medida, la incorporación de las propuestas de la población.

Las actuaciones participadas realizadas en esta fase fueron:

- Reuniones de análisis y priorización: en ellas se valoraron con la población las conclusiones del diagnóstico social y las líneas de trabajo propuestas, estableciendo a través de diferentes dinámicas aquellas que debían incorporarse al plan estratégico y el orden de prioridad en el que se deberían abordar. El equipo de dinamizadores redactó, a partir de los resultados, un primer borrador del plan.

- Consulta y participación en el plan: una nueva sesión de trabajo con la población sirve para presentar esta primera propuesta de plan estratégico y comprobar que se responde de forma adecuada a sus demandas, además de definir actuaciones concretas propuestas por los habitantes de cara al plan de acción.

- Redacción definitiva del plan estratégico/plan de acción: una vez redactado el borrador de los planes y aprobados inicialmente por el órgano gestor, estos documentos se someten a una amplia consulta pública, en la que fundamentalmente vía Internet se recogen las aportaciones de otras personas, instituciones y entidades interesadas. Los técnicos proceden a una redacción definitiva.

Una vez aprobado y en vigor el marco estratégico de la reserva, se produce el paso a la acción: la ejecución de las propuestas planteadas. El desarrollo de estas propuestas necesita, además de un amplio consenso entre la población, contar con una base participativa capaz de liderar los programas diseñados. La dinamización de esta base social es una tarea que, todavía hoy, supone un esfuerzo continuo tanto por parte del equipo técnico como de los participantes más implicados, tratando de compaginar resultados con eficiencia y aprovechar al máximo el esfuerzo que dedican los asistentes. La programación y ajuste conjunto de las convocatorias, reuniones, dinámicas y propuestas van tejiendo el hilo conductor del funcionamiento de la RBALE en estrecha simbiosis entre el equipo de gestión y los habitantes de Ancares.

- Constitución y puesta en marcha del Consejo de Participación 
Una de las primeras tareas que planteaba el plan de acción era la constitución de un órgano fijo capaz de mantener a largo plazo la participación de la población. La construcción de este grupo en un contexto de progresiva implicación local debía plantear un espacio común, para dotar a un grupo representativo, implicado y con conocimiento de causa de una estructura y un marco de funcionamiento adecuados que optimizasen el papel de la población dentro de la gestión de la reserva.

Para ello, la constitución del Consejo de Participación ha contado con su propio proceso, que a lo largo de varias sesiones y con diferentes dinámicas (como el Café del Mundo) consiguieron realizar las tareas necesarias: localizar agentes de interés, establecer sus funciones mínimas y consensuar un modelo de funcionamiento (número de miembros, representatividad, composición, frecuencia de las reuniones...).

Así, el 16 de mayo de 2012 se constituye formalmente el Consejo de Participación de la Reserva de la Biosfera y se aprueba su reglamento interno, que es ratificado a continuación por el órgano gestor de la RBALE e incluido en el plan estratégico. El Consejo ha trazado también su propia hoja de ruta, ajustada al plan de acción, y ha establecido el ámbito de sus relaciones con el resto de los órganos de la reserva de la biosfera, nombrando dos representantes que asisten a las reuniones del Consorcio y funcionan como interlocutores del mismo.

El Consejo de Participación, más allá de un papel consultivo (que también debe tenerlo), trabaja para desarrollar su amplia capacidad de decisión y actuación, estando detrás de varias propuestas concretas, por ejemplo en el ámbito turístico y ganadero. Este órgano es clave en la apuesta de la RBALE por introducir un modelo de gestión participada y por incluir a sus habitantes en las diferentes actuaciones.

El organigrama, composición y funcionamiento del Consejo de Participación se determinan en un reglamento de régimen interno elaborado por el propio Consejo y aprobado por el Consorcio, enmarcando las relaciones entre sus 22 miembros, que incluyen hosteleros, ganaderos, apicultores, asociaciones ecologistas, culturales, juveniles, de mujeres, artesanos, cazadores y pescadores y empresarios, entre otros.

\section{Segundo pilar: la comunidad científica}

Otro de los pilares básicos del funcionamiento de las reservas de la biosfera consiste en la investigación y la aplicación de los conocimientos científicos. El propio Plan de Acción de Madrid recoge, en la misión de las reservas de la biosfera, la necesidad de garantizar todos los aspectos de la sostenibilidad (medioambientales, económicos, sociales, espirituales y culturales). Esta garantía debe desarrollarse a través de fórmulas de integración 



Ángel Ruiz Mantecón (CSIC-ULe) con Octavio Cordero y José Antonio (presidente y socio de AGARBALE, respectivamente) compartiendo conocimientos | foto RBALE

del conocimiento incluyendo, por supuesto, el conocimiento científico, que debe liderar nuestra comprensión de las interacciones entre las personas y el resto de la naturaleza. Unir conocimiento científico y gestión participada es una estupenda vía de capacitación para mejorar nuestra intervención en los sistemas socio-ecológicos complejos, especialmente si somos capaces de potenciar el diálogo entre ciencia y política, la educación ambiental y la difusión multimedia de contenidos para la comunidad en general. Las reservas de la biosfera deben contribuir al desarrollo de modelos para la sostenibilidad mundial, nacional y local, y servir como lugares de aprendizaje donde los distintos actores, incluidas las comunidades científicas y de investigación, colaboren en la aplicación de los principios globales y locales del desarrollo sostenible.

El Plan Estratégico de la Reserva de la Biosfera de los Ancares Leoneses incorpora un Comité Científico destinado a guiar su funcionamiento en relación con el conocimiento y la investigación. Este Comité se constituyó en mayo de 2012 con la participación más que entusiasta de seis destacados miembros de la comunidad universitaria e investigadora castellano-leonesa.

Como no podía ser de otra manera dentro del contexto metodológico expuesto, la selección de académicos a ser invitados para conformar este grupo asesor tuvo como uno de los criterios clave la capacidad y apertura de interacción directa con la población así como la aceptación del papel propositivo de los habitantes de la zona bajo cualquier dinámica o grupo. La actividad del Comité ha sido sobresaliente en estos meses, especialmente debido
Equipo de la Universidad de Valladolid, liderado porAlipio García de Celis, en una ruta por Faro (Peranzanes) I foto RBALE 


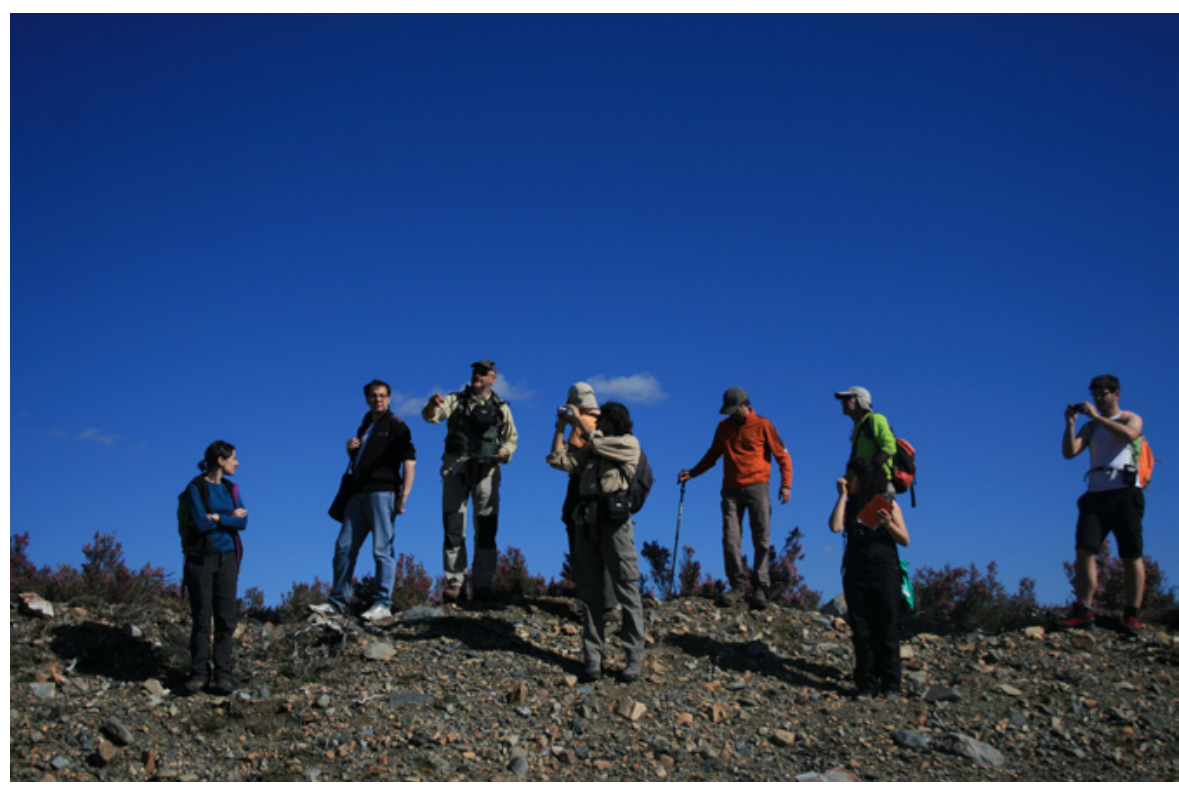

Un equipo de la Universidad de Valladolid investiga el patrimonio geológico de Ancares y sus posibilidades turísticas | foto RBALE

a que en un contexto muy restrictivo han sabido aportar una sabiduría, iniciativa y soporte que no se hubiera podido abordar sin contar con su apoyo incondicional.

El Comité Científico y el Consejo de Participación constituyen los órganos de carácter consultivo y de apoyo en la toma de decisiones de que dispone la reserva, sin perjuicio de que cada uno de ellos disponga de una gran capacidad de acción independiente, en el marco del modelo de gestión participada que trata de implantarse en la RBALE.

La complicidad y compromiso de ambos órganos, con la participación social como eje, se ha puesto de manifiesto en su primera propuesta de trabajo conjunto, que trata de establecer un modelo de intercambio permanente con los habitantes de los Ancares. Se trata de una serie de jornadas temáticas denominadas seminarios "Uniendo experiencias: ciencia y saber popular en los Ancares Leoneses". Una convocatoria periódica donde a través de charlas, foros, visitas y, en definitiva, encuentros y diálogos entre los científicos y la población local, se trata de compaginar la investigación con la cultura popular de los Ancares, traduciendo esta dialéctica en avances significativos de cara a la gestión del territorio. Los seminarios han trabajado sobre aspectos muy diferentes, como paisaje, turismo, ganadería extensiva, oficios tradicionales, cambio climático y aprovechamientos forestales.

En esta misma línea han surgido nuevos programas de trabajo científico en la reserva. El primero de ellos es el programa de asesoramiento directo a los 
ganaderos de extensivo que desarrolla Ángel Ruiz Mantecón (director de la estación Agrícola Experimental EAE -CSIC-) y, el segundo, el programa de Patrimonio Geomorfológico liderado por Alipio García de Celis, director del Departamento de Geografía de la Universidad de Valladolid.

Este programa, "Geodiversidad y Patrimonio Geomorfológico de la RBALE. Bases para su gestión, protección y aprovechamiento sostenible y propuesta de Rutas Turísticas", forma parte de un modelo pedagógico dirigido a la enseñanza universitaria, concretamente a la asignatura de Geomorfología práctica y aplicada del Grado en Geografía y Ordenación del Territorio, que hace hincapié en el desarrollo de habilidades profesionales en el alumnado.

El proyecto plantea una serie de actuaciones, que incluyen el reconocimiento y cartografía de la geodiversidad, el inventario de lugares de interés geomorfológico y la propuesta de rutas y materiales divulgativos orientados al turismo y al uso sostenible del patrimonio natural. Este proyecto se inició en el curso 2013 completando el trabajo del valle de Fornela, en Peranzanes, y se pretende continuar en los tres cursos posteriores, cada uno dedicado a otro de los municipios de la RBALE.

Según Alipio García, la filosofía que inspira este programa puede resumirse en unas pocas frases: El proyecto de geodiversidad se basa en conocer para apreciar, cuidar y aprovechar. Se pretende alcanzar un conocimiento lo más amplio posible del patrimonio geomorfológico de la RBALE, para sentar las bases de una correcta gestión sostenible del mismo, aprovechando su potencial turístico sin poner en peligro ni sus valores intrínsecos ni la continuidad de su aprovechamiento y disfrute por las generaciones futuras.

Estos proyectos, promovidos desde diferentes acuerdos y convenios con universidades y centros de investigación, son el avance de otros que se están gestando en la actualidad, a instancias de los órganos participados de la RBALE. Por ejemplo, una propuesta de recuperación del patrimonio inmaterial de la reserva y otra centrada en los efectos del cambio global sobre los servicios ambientales que prestan los ecosistemas de Ancares.

Finalmente, como broche del trabajo del Comité Científico, en esta ocasión bajo el paraguas de la Universidad de León, es la organización del curso de verano Espacios Naturales, Desarrollo Rural y Participación Social: Reservas de la Biosfera, dirigido por Estanislao de Luis Calabuig, catedrático de Ecología de la Universidad de León y presidente del propio Comité de la RBALE. Este curso, de formato itinerante, está dirigido a estudiantes, profesionales y personas interesadas en el modelo RBALE de participación social como herramienta de gestión territorial. Las ponencias, en varios casos, serán compartidas entre académicos y pobladores locales considerados expertos en su materia. 


\section{Tercer pilar: administracion local}

Finalmente, la RBALE cuenta con un órgano de gobierno de ámbito local que, entre otras funciones, sirve de nexo de unión entre instituciones y población. En este caso adopta la forma de consorcio. Bajo la denominación de Consorcio de la Reserva de la Biosfera de los Ancares Leoneses se constituye esta entidad en la que participan los ayuntamientos de Candín, Peranzanes, Vega de Espinareda, Villafranca del Bierzo y la Fundación Ciudad de la Energía.

El Consorcio de la RBALE asume la máxima responsabilidad administrativa en la configuración y gestión de la reserva, ya que es el representante directo de los territorios incluidos bajo esta denominación. Ostenta las escasas competencias políticas de la reserva y es el encargado de desarrollar, a nivel municipal, las determinaciones incluidas en los documentos de planificación y las propuestas que emanan de sus órganos de participación.

\section{DESARROLLO DEL PLAN DE ACCIÓN}

El verdadero reto, lo obvio y al mismo tiempo lo dificultoso de este modelo consiste, por supuesto, en pasar del papel a la acción. Hacer realidad lo planificado en su momento, avanzar en actuaciones verdaderamente transformadoras y asumidas por la población como propias es, con mucho, la tarea más complicada de la gestión de la RBALE; una tarea en la que no ayuda nada la actual escasez de recursos ni la falta de perspectiva que muestran sin pudor muchas de las administraciones responsables.

No obstante, la reserva, poco a poco, con tropiezos y dificultades, con éxitos y fracasos, va consolidando una línea de acción, quizá insignificante de momento en comparación con otros proyectos pero a cambio fuertemente enraizada en el territorio y en sus habitantes, o eso al menos es lo que estamos intentando. Los siguientes apartados muestran algunas de las tareas concretas que se han ido desarrollando con los sectores más representativos de la población ancareña.

\section{Empezando a rodar}

Algunas cuestiones importantes del trabajo de la reserva de la biosfera se encuentran aún en sus fases iniciales, trabajando duro para sacarlas adelante. Entre ellas se puede citar la potenciación del sector turístico o la puesta en marcha de un plan de comunicación (incluyendo una profunda renovación del portal web que debe convertirse en una herramienta más de apoyo al trabajo interactivo). 

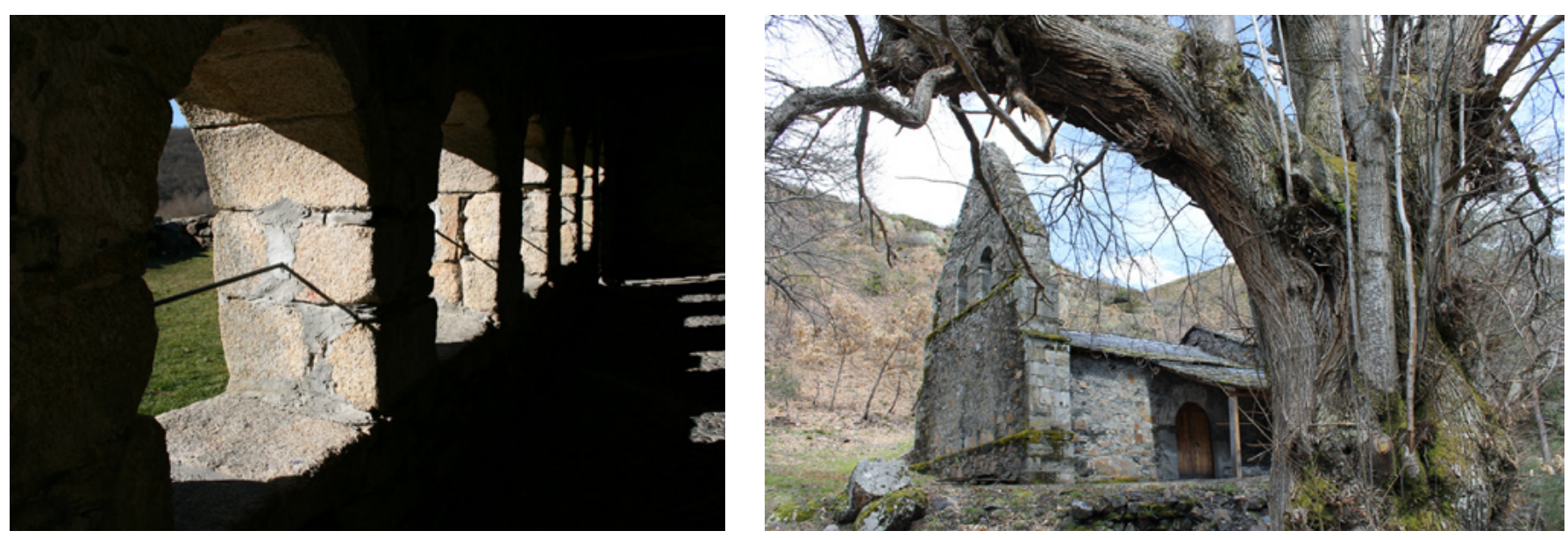

Suárbol | foto RBALE

También se han iniciado otras líneas de trabajo, como la colaboración con el Proyecto Ríos El Bierzo-Laciana (proyecto de educación, formación y participación ambiental alrededor de los sistemas fluviales), el diseño de un proyecto participado para el desarrollo de la apicultura o la incorporación de la RBALE a la Mesa del Castaño que debe responder a las demandas locales sobre la conservación del patrimonio natural.

Aún queda mucho trabajo por hacer en este sentido, y ojalá los recursos y el tiempo vayan dando más respuestas a estos problemas. Igualmente, el trabajo sobre el patrimonio inmaterial, donde la población tiene todo por decir, es uno de los retos de futuro para la RBALE.

Pero, hay proyectos que ya llevan un largo camino recorrido y se encuentran en pleno proceso de maduración. Destaca, entre ellos, el trabajo que se ha ido desarrollando sobre la ganadería extensiva que, por ser el primero y por contar con un núcleo de participación más amplio, ha ido consiguiendo algunos logros sustanciales.

\section{La importancia de los ganaderos en la reserva de la biosfera}

El diagnóstico social de la RBALE puso de manifiesto enseguida la inquietud y la voluntad de los ganaderos de extensivo de la zona por aunar esfuerzos ante las adversidades que el sector vive actualmente, como son la subida de precios de piensos y combustible, la falta de representatividad ante la administración, la falta de canales de comercialización cortos o la baja consideración de la sociedad hacia la actividad y sus profesionales.

Y, en cambio, la ganadería extensiva es un elemento clave en la conservación de los paisajes de alto valor natural de toda la Unión Europea y, especialmente, de sistemas de montaña como Ancares. La ganadería 


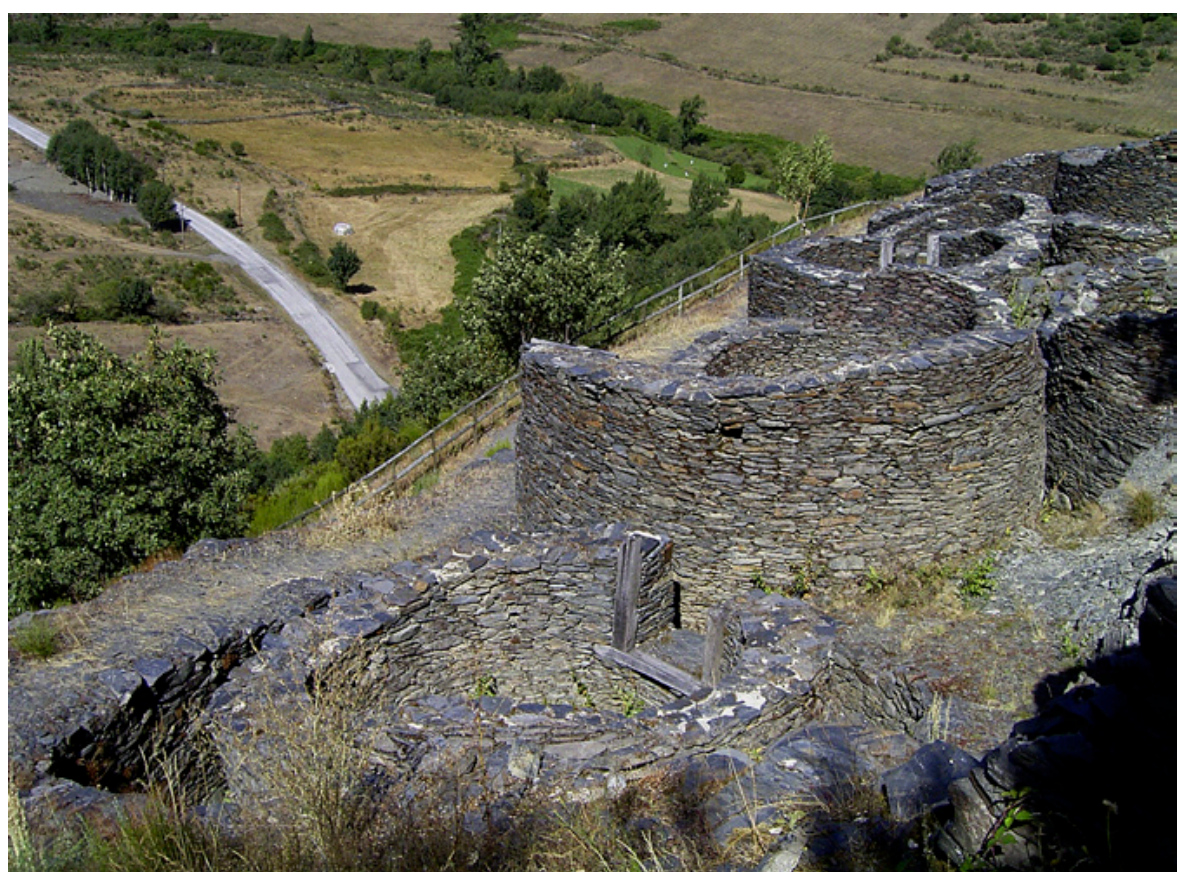

Castro de Chano Peranzanes, agosto de 2006 | foto José M. a Rodrigo Cámara

contribuye al mantenimiento de la biodiversidad y el paisaje, previene los incendios forestales, mantiene los caminos y las cañadas y aporta también un patrimonio cultural y natural de gran calado. Se puede afirmar, sin temor a equivocación, que los ganaderos han sido los pastores del paisaje ancareño desde tiempos inmemoriales y que el mantenimiento y la sostenibilidad de su labor debe ser una prioridad en cualquier modelo de gestión del patrimonio que pretenda conservar sus valores y procesos.

Es por ello que el fortalecimiento del sector ganadero de extensivo se convirtió en una prioridad inmediata de cara al trabajo en la RBALE. En diciembre de 2011, once ganaderos de extensivo firmaban el acta fundacional de la Asociación de Ganaderos de la RBALE (AGARBALE), constituida con el respaldo técnico de la propia reserva de la biosfera.

El proceso de constitución se desarrolló a través de varias reuniones de trabajo donde los ganaderos concretaron los objetivos de la asociación y su regulación, volcándolos en los estatutos de la nueva organización. Entre sus fines destaca su idea de servir como interlocutor válido del sector local ante las diferentes administraciones y entidades públicas, y ante las empresas que desarrollan actividades agrarias. Los ganaderos también se plantean, de cara al futuro, la creación de una imagen que identifique los productos ganaderos de la RBALE (ya han elaborado sus primeros materiales en este sentido), asesorar y agilizar los trámites burocráticos de 


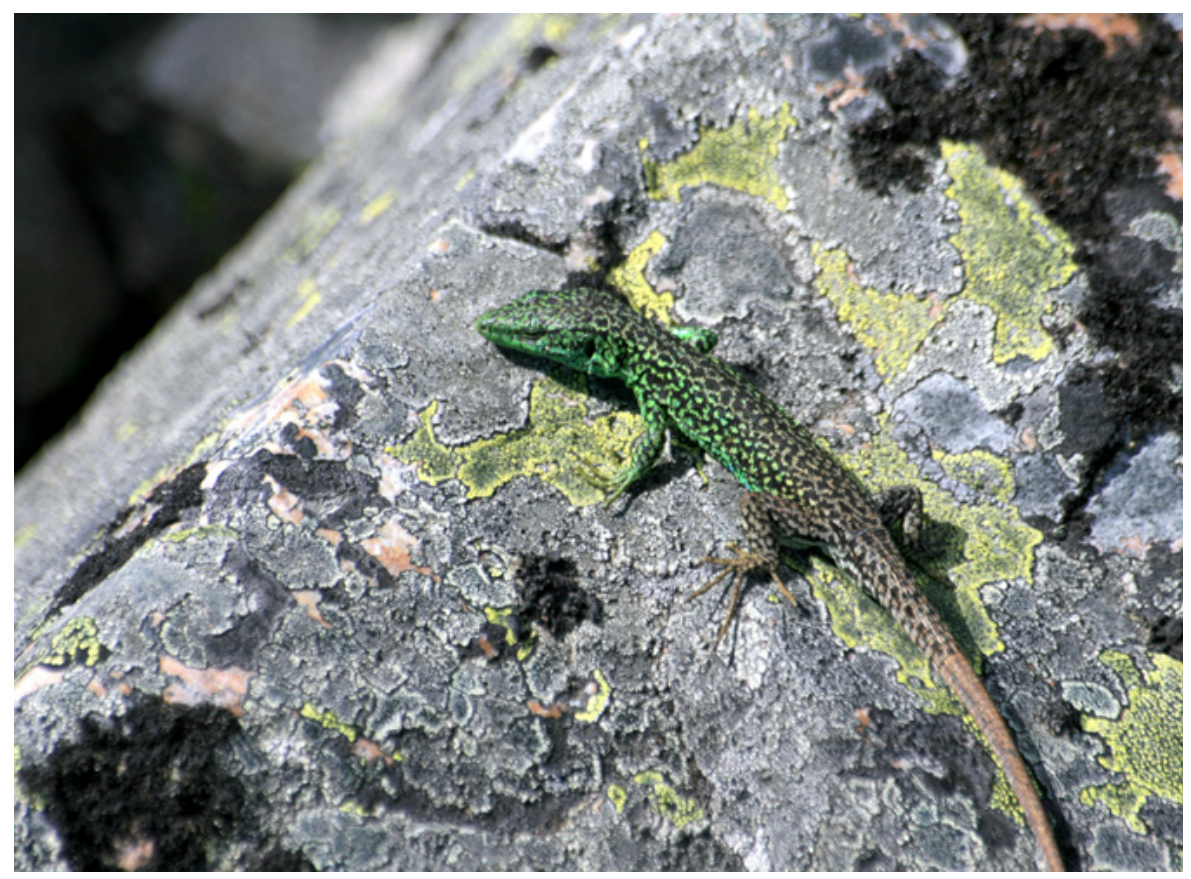

Un ejemplar de lagartija serrana, endémica del norte ibérico, toma el sol en uno de los numerosos canchales de las cumbres de Ancares | foto RBALE

sus asociados y también potenciar su propio tejido organizativo. No obstante, su tarea más importante consiste en revalorizar la ganadería extensiva y hacer patente el valor intrínseco que tiene para el mantenimiento del entorno.

Para ello han comenzado a trabajar con Ángel Ruiz Mantecón, catedrático de Veterinaria y miembro del Comité Científico de la RBALE, quien les asesora en cuestiones relativas a la mejora de las instalaciones, el manejo del ganado en extensivo, la nutrición y la incorporación de productos locales a sus explotaciones.

Otro hito importante ha sido la organización de un encuentro de ganadería extensiva a nivel regional, que ha servido como ámbito de debate entre todos los ganaderos extensivos de Castilla y León, representantes de un modelo de gestión del territorio injustamente olvidado pero con un activo papel en la conservación del patrimonio natural y cultural. Finalmente, la Asociación de Ganaderos de la RBALE, en el marco del programa LIFE+ de Conservación del Urogallo Cantábrico que desarrolla la Fundación Biodiversidad, participa en el establecimiento de acuerdos de custodia del territorio para la conservación del hábitat del urogallo cantábrico: una forma de poner en valor la labor de conservación que la actividad ganadera realiza en muchos de nuestros ecosistemas. 


\section{EL PRESENTE, EL FUTURO}

El trabajo de estos dos últimos años ha sido sin duda duro y exigente, tanto para la gerencia (que ha afrontado los pasados doce meses y el futuro en solitario debido a la pérdida de ayudas y financiación), los técnicos y la asistencia externa como, sobre todo, para la población, que se ha implicado en la gestión de la reserva sin demandar ni esperar apenas nada a cambio.

La Reserva de la Biosfera de los Ancares Leoneses ya cuenta con su hoja de ruta, sus órganos de funcionamiento y su plan de acción fruto de todo este trabajo y de la implicación de sus habitantes. $Y$ aunque estos planes necesitan fondos y apoyos económicos para su realización (que el equipo técnico de la RBALE, inasequible al desaliento, se encarga de solicitar a las diferentes instituciones), y aunque corren tiempos difíciles, hay un soporte que nunca falla: el que proporciona una población organizada, informada y sensibilizada que apuesta por un modelo en el que las decisiones y la orientación del trabajo recaen directamente sobre ellos. Los protagonistas de esta tarea formidable son los auténticos herederos de un patrimonio que una vez fue considerado tan excepcional como para ser declarado reserva de la biosfera.

La dedicación, tiempo y energía invertida nada tiene que ver con el diseño de actividades a golpe de teclado y la inundación de los usuarios con publicidad de reclamo. Sin embargo, la situación actual de extrema escasez de recursos limita fuertemente la capacidad técnica para mantener estos procesos con un nivel mínimo de calidad y garantizar una participación adecuada. Muchas propuestas interesantes están tan mal dotadas, o el desarrollo de estas debe dilatarse tanto en el tiempo, que es difícil mantener la ilusión y las ganas en ello.

Vivimos en un mundo individualista y con un sistema de representación cuestionado en los últimos tiempos por muchos sectores de la sociedad. La reivindicación de implicarse en la toma de decisiones está cada vez de forma más audible en los medios y la calle. ¿Tenemos realmente la capacidad, como ciudadanos, de hacerlo? ¿Somos capaces de liderar nuestro futuro? ¿Se puede participar realmente en la gestión del patrimonio común o en el desarrollo sostenible desde una perspectiva ciudadana? Estas preguntas que se plantean en cada ciudad y cada pueblo de nuestro país suponen un reto para los territorios que, como las reservas de la biosfera, constituyen el banco de pruebas de las nuevas estrategias de desarrollo y conservación del patrimonio.

La RBALE se plantea, también, una dimensión internacional de intercambio de experiencias y perspectiva, especialmente con las reservas de Canadá. Hace unos meses, compartimos con Stanley Boychuk, presidente del Comité 
MaB Canadiense, nuestra manera de funcionar, sorprendentemente alineada con el trabajo realizado por las reservas de la biosfera de su país junto con las Primeras Naciones canadienses. La apuesta por la unión del mundo académico con la sabiduría popular y la utilización del capital social como potencial de desarrollo del territorio parece algo no exclusivo de la montaña del noroeste leonés, sino una visión global que acompaña quizás a los cambios que el mundo está viviendo en esta época de crisis económica y ambiental.

El acceso de los ciudadanos a la toma de decisiones en la gestión de su territorio y la conservación de su patrimonio es el reto de futuro al que se enfrenta la Reserva de la Biosfera de los Ancares Leoneses. Los pasos que se han dado hasta ahora muestran que no se trata de una quimera sino de algo que puede acercarse mucho a lo cotidiano, al papel activo de cualquier ciudadano con respecto a su entorno. Las propuestas y experiencias puestas en marcha en la RBALE muestran un resultado alentador, cuando la ciudadanía dispone de espacios adecuados y puede acceder a la formación y a las capacidades necesarias puede implicarse y participar de una gestión que le atañe directamente y obtener resultados satisfactorios. El desafío al que se enfrenta la RBALE en su futuro inmediato consiste en profundizar y progresar en esta tarea, consolidando el camino ya recorrido y encauzando nuevas ideas e iniciativas en su papel como laboratorio de participación.

\section{Más información:}

www.ancaresleoneses.es

info@ancaresleoneses.es

Sobre reservas de la biosfera:

www.unesco.org/new/en/natural-sciences/environment/ecological-sciences/ man-and-biosphere-programme/

Programa MaB en España:

www.magrama.gob.es/es/parques-nacionales-oapn/reservas-biosfera/

\section{Nota de la redacción revista ph}

Agradecemos a las siguientes personas su colaboración en esta sección aportando imágenes de los Ancares Leoneses que fueron publicadas en el perfil de Facebook del IAPH entre los meses de abril y junio de 2013:

Fernando Carrera Ramírez | Rodrigo Garnelo Merayo | Javier Pérez Gil | José M. ${ }^{a}$ Rodrigo Cámara 